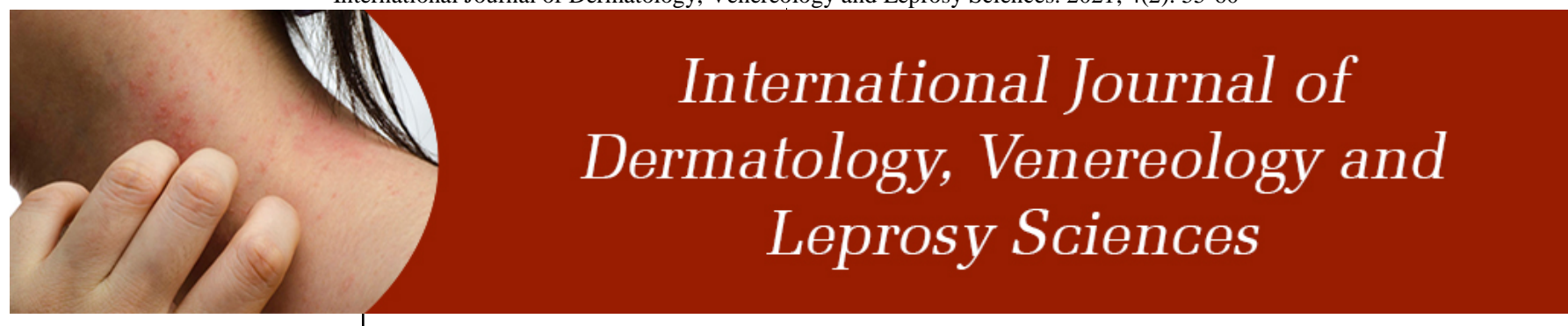

E-ISSN: 2664-942X P-ISSN: 2664-9411 www.dermatologypaper.com Derma 2021; 4(2): 55-60 Received: 08-07-2021 Accepted: 10-08-2021

Dr. Neha Saraswat Junior Resident, Department of D.V.L. Maharishi Markandeshwar Medical College \& Hospital, Solan, Himachal Pradesh, India

Dr. Rajwinder Singh Professor, Department of D.V.L. Maharishi Markandeshwar Medical College \& Hospital, Solan, Himachal Pradesh, India

Dr. Vinay Shanker Professor and H.O.D. Department of D.V.L. Maharishi Markandeshwar Medical College \& Hospital, Solan, Himachal Pradesh, India

Corresponding Author: Dr. Neha Saraswat Junior Resident, Department of D.V.L. Maharishi Markandeshwar Medical College \& Hospital, Solan, Himachal Pradesh, India

\section{Assessment of visual analogue scale (VAS) in patients undergoing elective hair reduction with diode laser}

\author{
Dr. Neha Saraswat, Dr. Rajwinder Singh and Dr. Vinay Shanker
}

DOI: https://doi.org/10.33545/26649411.2021.v4.i2a.94

\begin{abstract}
Background: Diode lasers are the most popular for hair removal due to the deep penetration and targeting of the hair follicle. Our study aimed at improvement in visual analogue scale (VAS), in patients undergoing elective hair reduction with diode laser.

Aim: Assessment of visual analogue scale at every sitting.

Methodology: this prospective observational study was undertaken in the department of dermatology \& venereology and leprosy (DVL), Maharishi Markandeshwar Medical College and Hospital, Kumarhatti, Solan during the Dec 2019 to Dec 2020. Total of 30 patients aged more than 16yrs fulfilling inclusion criteria were included in the present study. Lumenis Light Sheer Desire Laser System ${ }^{\circledR}$ of $805 \mathrm{~nm}$ was used in treatment.

Result: In present study, total of 30 patients fulfilling inclusion criteria were included in the study after obtaining the informed consent. All the patients who participated in study were females. There was a significant higher mean of visual analogue score among the patients compared to the $1^{\text {st }}$ sitting. $(p<0.05)$

Conclusion: There was a significant improvement in the mean score of VAS among the patients at $5^{\text {th }}$ sitting compared to the $1^{\text {st }}$ sitting. The Diode laser technique is efficient and safe in patients undergoing the elective hair reduction.
\end{abstract}

Keywords: diode laser and visual analogue scale.

\section{Introduction}

Diode lasers are the most popular for hair removal due to the deep penetration and targeting of the hair follicle. Often referred to as the gold standard in hair removal, the diode laser $(810 \mathrm{~nm})$ is highly reliable, has high papilla absorption, and can address a wide range of skin types, including skin types V and VI.(1) Diode lasers use the principle of selective photo thermolysis to target specific chromophores in the skin, usually melanin or blood. The lasers damage the chromophores by selective heating, without damaging the surrounding tissue. Melanin is the chromophore in the hair follicles, which is targeted and damaged, which results in disrupted growth and regeneration of hair. Our study has evaluated Subject satisfaction with visual analogue scale (VAS) from 0 for complete dissatisfaction to 10 for complete satisfaction.

\section{Material and Method}

The present study was undertaken in the department of dermatology \& venerology and leprosy (DVL), Maharishi Markandeshwar Medical College and Hospital, Kumarhatti, Solan.

Study Design: Prospective Observational Study.

Duration: December 2019 to December 2020

\section{Selection of patients}

All the patients, who came to the Out Patient Department of Dermatology, Venereology and leprosy of MMMC\&H, for the first time, for elective hair reduction with diode laser.

Sample size: Minimum sample size of 30 .

Inclusion criteria

Age $>16 y r s$ 


\section{Exclusion criteria}

- History of previous laser hair removal treatment

- Current or past history of skin cancer

- Severe concurrent medical conditions

- Any active cutaneous eruption in the treatment area

\section{Procedures:}

\section{Preparation:}

1. Detailed clinical history to rule out infections, drug intake, etc.

2. In patients of hirsutism modified Farriman-Gallwey score was calculated, along with following investigations to rule out the cause and treated accordingly

- Serum testosterone

- Serum prolactin

- USG pelvis

- $\quad$ Serum T3, T4 and TSH

3. Avoid hair removal by plucking, bleaching or waxing for 6 weeks before the procedure.

4. Sunscreens were prescribed 4 to 6 weeks before the procedure, especially on exposed areas.

5. Count number of hairs in per $\mathrm{cm}^{2}$ area

6 . Shave the area before the procedure.

7. Obtain an informed consent.

8. Peri-follicular oedema and erythema is an expected side effect, and this was explained to the patient.

\section{Equipment}

Lumenis Light Sheer Desire Laser System ${ }^{\circledR}$ OF 805nm, used in Dermatology, Venereology and Leprosy department of MMMCH, Kumarhatti, Solan.

\section{Technique:}

1. Clean the area to remove oils from the skin

2. Position the patient comfortably.

3. Use eye protection for both the operator as well as the patient

4. Apply ice pack on area to be treated

5. Place the handpiece (XC or HS tip), perpendicular to the skin according to area to be treated. Ensure that it is pressed down gently and firmly to bring the follicles close to the surface and temporarily decrease the blood supply to the local area.

6. A total of 5 sittings were given at an interval of 4 to 6 weeks.

\section{Post procedure care}

1. Although most machines have inbuilt cooling systems, ice packs or cooling agents was used post-procedure to minimize pain and swelling.

2. In case of inadvertent epidermal injury, topical antibiotics was used.
3. Strict sun protection and the use of sunscreens.

4. Topical steroid creams were prescribed, if there were any side effects like erythema and swelling.

Pulse width and fluence were used as per the manufacturer's guidelines for skin type IV and V For XC tip:

\begin{tabular}{|c|c|c|}
\hline Sitting & Pulse Width (Ms) & Fluence (J/Cm2) \\
\hline First & 100 & $18-20$ \\
\hline Second & 100 & $20-22$ \\
\hline Third & 100 & $22-24$ \\
\hline Fourth & 30 & $20-22$ \\
\hline Fifth & 30 & $22-24$ \\
\hline
\end{tabular}

For HS tip

\begin{tabular}{|c|c|c|}
\hline Sitting & Pulse width (ms) & Fluence $(\mathrm{j} / \mathrm{cm} 2)$ \\
\hline first & 100 & $5.0-5.5$ \\
\hline second & 100 & $5.5-6.0$ \\
\hline third & 30 & $5.0-5.5$ \\
\hline fourth & 30 & $5.5-6.0$ \\
\hline fifth & 30 & $6.0-6.5$ \\
\hline
\end{tabular}

Ethical consideration: Informed and written consent (in the language they best understand) was taken from each subject before collecting data and blood sample. Only those individuals, who volunteer to participate in the study, were included and the data was kept confidential. The study did not impose any burden on the subjects and the Institute; therefore, the study is ethically justified.

\section{Results}

In present study, total of 30 patients fulfilling inclusion criteria were included in the study after obtaining the informed consent. All the patients who participated were females, as males are not as concerned as females about their hairs. The mean age of the patients was found to be $27.83 \pm 6.53 y$ rs of age, with majority of patients in group of $17-25 y r s$ (40\%) followed by $26-30 y r s$ of age (33.3\%).

Table 1: Mean age of the patients in present study

\begin{tabular}{|c|c|c|c|c|c|}
\hline & N & Minimum & Maximum & Mean & SD \\
\hline Age in yrs & 30 & 17 & 43 & 27.66 & 6.47 \\
\hline
\end{tabular}

Out of total 30 patients, minimum age of the patient was $17 \mathrm{yrs}$ and maximum age was $43 y r s$. Mean age of the patients was found to be $27.66 \pm 6.47 \mathrm{yrs}$

Table 2: Showing the age wise distribution of patients

\begin{tabular}{|c|c|c|}
\hline & Frequency & Percent \\
\hline 17-25yrs & 12 & 40 \\
\hline 26-30yrs & 10 & 33.3 \\
\hline >31yrs & 8 & 26.7 \\
\hline
\end{tabular}

Majority of patients in group of 17-25yrs (40\%), followed by 26$30 \mathrm{yrs}$ of age (33.3\%) and $>30 \mathrm{yrs}$ in $26.7 \%$. 


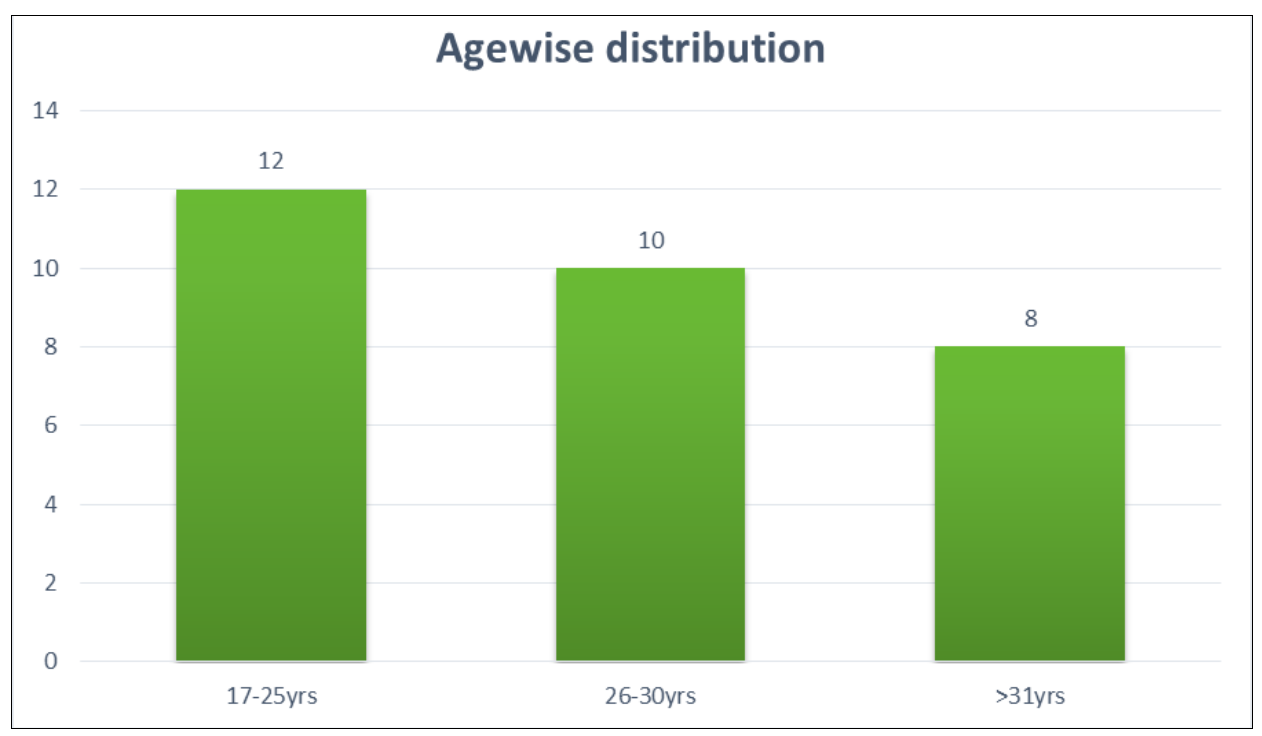

Fig 1: Showing the age wise distribution of patients

Out of 30 patients, 12 patients were between the age of 17-25yrs, 10 patients between 26-30yrs and 8 patients were of age $>31 \mathrm{yrs}$.

Table 3: Showing the mean level of visual analogue scale score during various sitting

\begin{tabular}{|c|c|c|c|c|c|}
\hline & $\mathbf{N}$ & Minimum & Maximum & Mean & SD \\
\hline 1st sitting Visual analogue scale & 30 & .0 & .0 & .000 & .00 \\
\hline 2nd sitting Visual analogue scale & 30 & 1.0 & 5.0 & 2.00 & 1.01 \\
\hline 3rd sitting Visual analogue scale & 30 & 1.0 & 6.0 & 3.70 & 1.34 \\
\hline 4th sitting Visual analogue scale & 30 & 2.0 & 8.0 & 5.46 & 1.35 \\
\hline 5th sitting Visual analogue scale & 30 & 2.0 & 9.0 & 6.36 & 1.51 \\
\hline
\end{tabular}

Subject satisfaction was assessed using visual analogue scale (VAS) from 0 for complete dissatisfaction to 10 for complete satisfaction. Mean VAS at $1^{\text {st }}$ sitting; 0 , $2^{\text {nd }}$ sitting; 2.0 , $3^{\text {rd }}$ sitting; $3.70,4^{\text {th }}$ sitting; $5.46,5^{\text {th }}$ sitting; 6.36 .

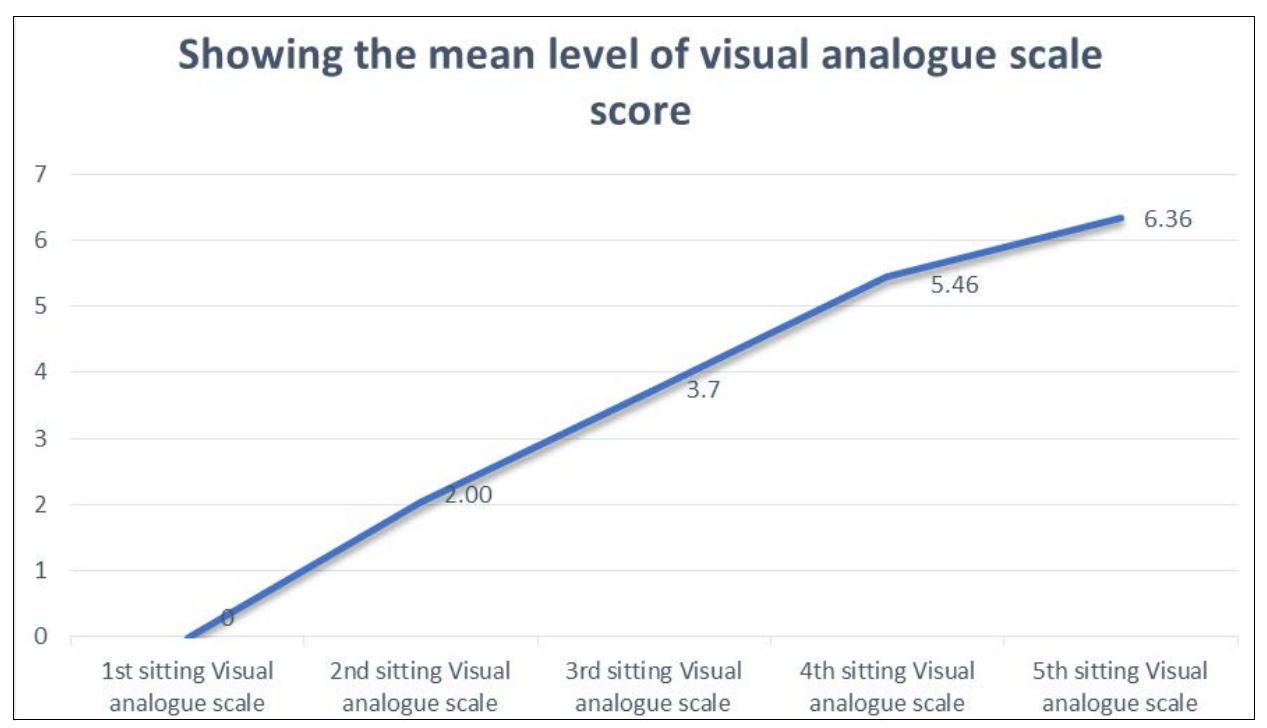

Fig 2: Showing the mean level of visual analogue scale score

VAS increasing from 0 at $1^{\text {st }}$ sitting to 6.36 at $5^{\text {th }}$ sitting

Table 4: Showing the mean level of hair density during various sitting

\begin{tabular}{|c|c|c|c|c|c|}
\hline & N & Minimum & Maximum & Mean & SD \\
\hline 1st sitting Hair Density & 30 & 3.0 & 44.0 & 17.33 & 10.41 \\
\hline 2nd sitting Hair Density & 30 & 3.0 & 37.0 & 13.86 & 8.75 \\
\hline 3rd sitting Hair Density & 30 & 1.0 & 30.0 & 11.06 & 7.74 \\
\hline 4th sitting Hair Density & 30 & 1.0 & 22.0 & 8.26 & 5.75 \\
\hline 5th sitting Hair Density & 30 & 1.0 & 20.0 & 6.90 & 4.97 \\
\hline
\end{tabular}

Hair density was measured by drawing $1 \times 1 \mathrm{~cm}^{2}$ box and calculating the terminal hairs in that box. Mean hair density at $1^{\text {st }}$ sitting; $17.33,2^{\text {nd }}$ sitting; $13.86,3^{\text {rd }}$ sitting; $11.06,4^{\text {th }}$ sitting; $8.26,5^{\text {th }}$ sitting; 6.90 . 


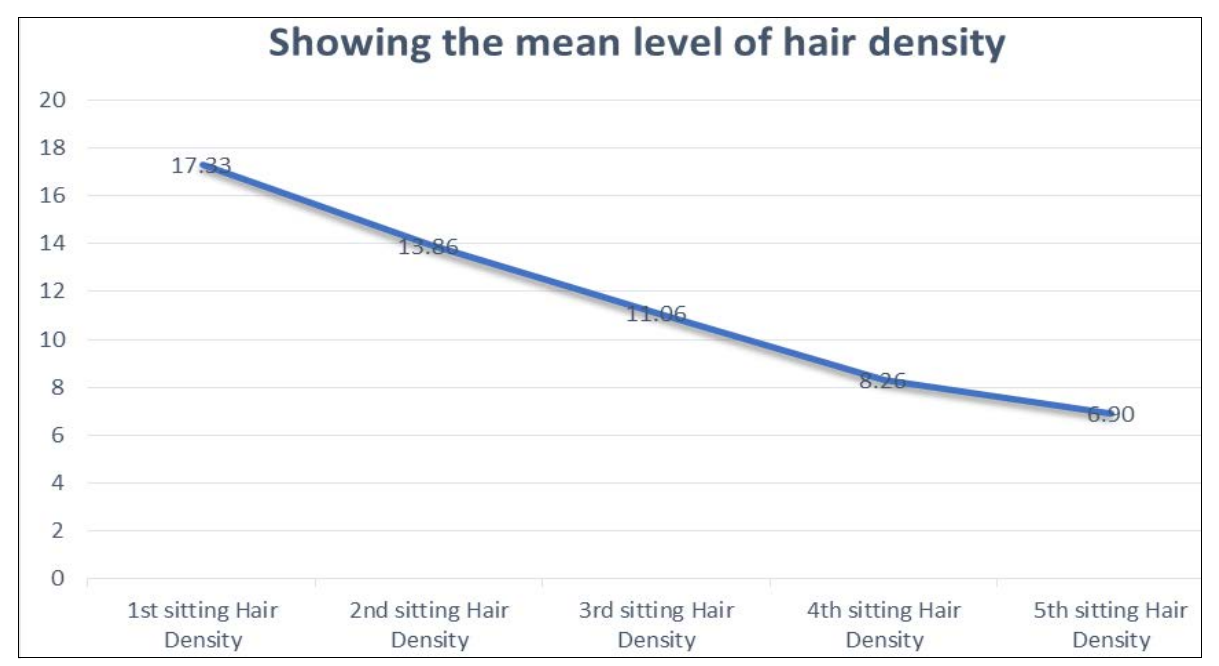

Fig 3: Showing the mean level of hair density

Mean hair density decreasing from 17.33 at $1^{\text {st }}$ sitting to 6.90 at $5^{\text {th }}$ sitting

Table 5: Comparison of mean visual analogue scale score at various sittings using paired t-test

\begin{tabular}{|c|c|c|c|c|}
\hline \multicolumn{5}{|c|}{ Paired Samples Statistics } \\
\hline & & Mean & SD & Paired t-test (p-value) \\
\hline \multirow{2}{*}{ Pair 1} & 1st sitting Visual analogue scale & .00 & .00 & \multirow{2}{*}{$<0.0001$} \\
\hline & 2nd sitting Visual analogue scale & 2.00 & 1.02 & \\
\hline \multirow{2}{*}{ Pair 2} & 1st sitting Visual analogue scale & .00 & .00 & \multirow{2}{*}{$<0.0001$} \\
\hline & 3rd sitting Visual analogue scale & 3.70 & 1.34 & \\
\hline \multirow{2}{*}{ Pair 3} & 1st sitting Visual analogue scale & .00 & .00 & \multirow{2}{*}{$<0.0001$} \\
\hline & 4th sitting Visual analogue scale & 5.47 & 1.36 & \\
\hline \multirow{2}{*}{ Pair 4} & 1st sitting Visual analogue scale & .00 & .00 & \multirow{2}{*}{$<0.0001$} \\
\hline & 5th sitting Visual analogue scale & 6.37 & 1.52 & \\
\hline
\end{tabular}

$\mathrm{P}$ value was highly significant with every subsequent sitting on comparing with first sitting.

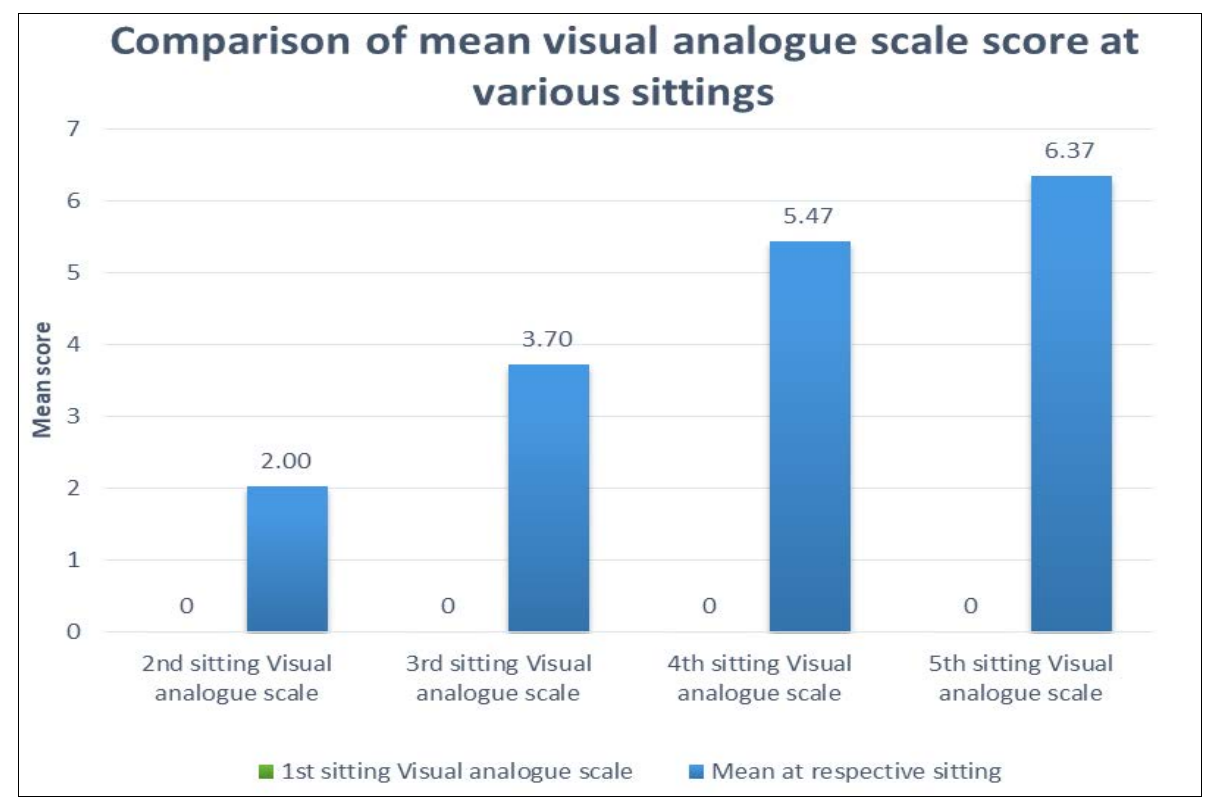

Fig 4: Comparison of mean visual analogue scale score at various sittings

Table 6: Comparison of mean hair density at various sittings using paired t-test

\begin{tabular}{|c|c|c|c|c|}
\hline & & Mean & SD & Paired t-test (p-value) \\
\hline \multirow{2}{*}{ Pair 1} & 1st sitting Hair Density & 17.33 & 10.42 & \multirow{2}{*}{$<0.0001$} \\
\hline & 2nd sitting Hair Density & 13.87 & 8.75 & \\
\hline \multirow{2}{*}{ Pair 2} & 1st sitting Hair Density & 17.33 & 10.42 & \multirow{2}{*}{$<0.0001$} \\
\hline & 3rd sitting Hair Density & 11.07 & 7.75 & \\
\hline \multirow{2}{*}{ Pair 3} & 1st sitting Hair Density & 17.33 & 10.42 & \multirow{2}{*}{$<0.0001$} \\
\hline & 4th sitting Hair Density & 8.27 & 5.75 & \\
\hline \multirow{2}{*}{ Pair 4} & 1st sitting Hair Density & 17.33 & 10.42 & \multirow{2}{*}{$<0.0001$} \\
\hline & 5th sitting Hair Density & 6.90 & 4.98 & \\
\hline
\end{tabular}

$\mathrm{P}$ value was highly significant with every subsequent sitting on comparing with first sitting. 


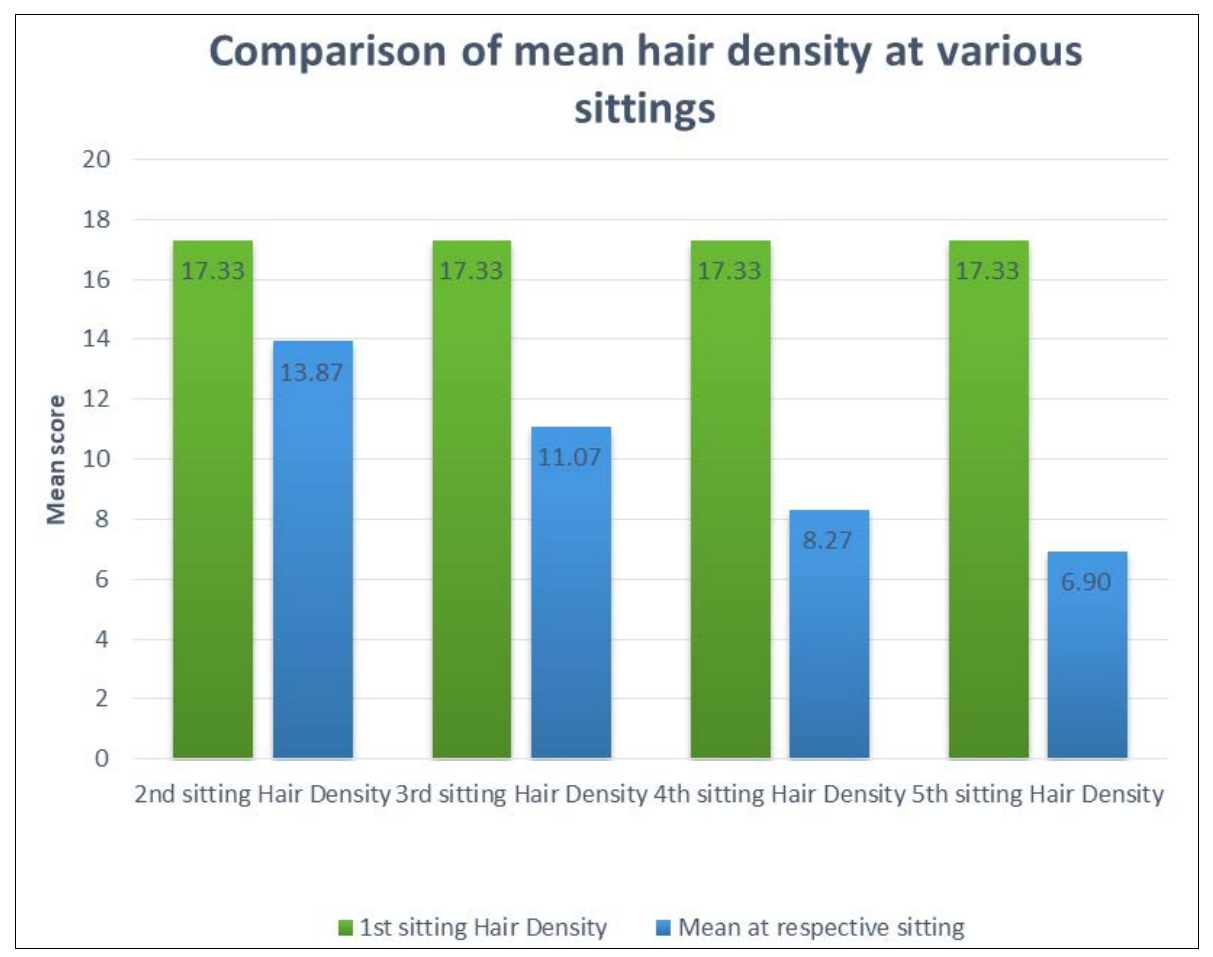

Fig 5: Comparison of mean hair density at various sittings

\section{Discussion}

Unwanted facial hair (UFH) is an important issue: over $40 \%$ of the women in the general population have some degree of UFH, ranging from nearly invisible growth to extensive growth. It has psychological and psychosocial impact, independent from the severity, which should not be underestimated. Hirsutism is the most common cause of unwanted facial hairs, which is defined as the presence of excessive terminal hair in females in androgen dependent areas.

The diode laser system generates a laser light of $\sim 800 \mathrm{~nm}$ wavelength. In a prospective controlled trial, where they used this laser, resulted in significant reduction of hair growth. Overall, clinical studies with the diode laser for hair reduction have reported variable success rates, ranging from $65-75 \%$ hair reduction at 3 months after one to two treatments with fluences of $10-40 \mathrm{~J} / \mathrm{cm} 2$ to $>75 \%$ hair reduction in $91 \%$ of subjects 8 months after three to four treatments at $40 \mathrm{~J} / \mathrm{cm}$.

Alternatively, conflicting data exists that, whether the fluence of the laser, should be between $15-40 \mathrm{~J} / \mathrm{cm} 2$ for therapeutic success. In darker skin, less fluence may be required to attain the same efficacy. In general, the diode lasers are found to be better tolerated by people of darker skin types (V-VI) than the ruby laser; because the energy absorbed by the melanin is $\sim 30 \%$ less than that of ruby laser, but has a better optical penetration. Although the incidence of pigmentary skin changes has been as high as $29 \%$, most of these changes are transient and well tolerated. Advantages of diode laser are as follows:

- The Diode laser features a longer wavelength and thus able to provide better results among dark-skinned people.

- This laser provides safer and deeper penetration into the skin layer.

- Large areas of the body tend to recover faster.

Treatment with diode laser is effective on medium to dark hair on any skin type, when the hair is in its anagen phase of growth; because of this reason, multiple sessions at an interval of one month are required for the treatment, as at a time not all the hairs are in anagen phase of growth. Therefore; the number of sessions required for the hair reduction varies.

The mean age of patients in the present study was $27.66 \pm 6.47 y r s$. Similar to present study, Baig $\mathrm{T}$ et al., also documented the mean age of participants to be $26.12 \pm 5.83$ years. (2). In an another study women were between the ages 24 and 41 years with a mean age of 32.27 \pm 4.21 years, which is slightly higher than our study.(3)

VAS is a subject satisfaction score; with each sitting, there was a significant higher mean of visual analogue score among the patients compared to the $1^{\text {st }}$ sitting. $(p<0.05)$ The mean VAS score increased from $2.00 \pm 1.01$ at $2^{\text {nd }}$ sitting, $3.70 \pm 1.34$ at $3^{\text {rd }}$ sitting, $5.46 \pm 1.35$ at $4^{\text {th }}$ sitting and $6.36 \pm 1.51$ at $5^{\text {th }}$ sitting. Similar to our study ayatollahi et al observed increase in VAS from 0 to 5.07 after diode laser hair reduction ${ }^{[4]}$.

With the treatment, hair density was found to reduce significantly at each sitting compared to the mean of $1^{\text {st }}$ sitting. $(p<0.05)$ The mean level reduced from $17.33 \pm 10.41$ at $1^{\text {st }}$ sitting, $13.86 \pm 8.75$ at $2^{\text {nd }}$ sitting, $11.06 \pm 7.74$ at $3^{\text {rd }}$ sitting, $8.26 \pm 5.75$ at $4^{\text {th }}$ sitting and $6.90 \pm 4.97$ at $5^{\text {th }}$ sitting. In a study conducted over 14 female patients, calculated hair density within $2 \times 2 \mathrm{~cm}^{2}$ area. Average hair density at baseline was 69.1, which came down to 20.6 at 6 months ${ }^{[5]}$. HR-AL hamamy observed hair density to be $21.58 \pm 11.59$ at baseline, $15.04 \pm 9.17$ after $1^{\text {st }}$ session, $11.77 \pm 7.55$ after $2^{\text {nd }}$ session, 8.96 \pm 6.77 after $3^{\text {rd }}$ session ${ }^{[6]}$. Similarly, in a study over 8 patients, approximate decrease in hair growth was from $75 \%-90 \%{ }^{[7]}$. In a study conducted over people of various ethnicity, per $\mathrm{cm} 2$ reduction of hair was noticed, 92.5\% for Asians, 81.5\% for people with black skin,86.9\% for mixed race and $88.2 \%$ for people of white coloured skin ${ }^{\text {[8] }}$. Zhou et al calculated hair density by drawing a circle of $3.5 \mathrm{~mm}$ and calculating the hairs within the circle. Reduction rate of hair growth noticed was $72 \pm 18$ at the end of 3 
months ${ }^{[3]}$.

Most of the patients had erythema and peri-follicular edema, which was transient. $1^{\text {st }}$ degree burn was noticed in one $(0.03 \%)$ patient at chin on $2^{\text {nd }}$ sitting, and was given topical anti-inflammatory.

\section{Conclusion}

This prospective observational clinical study of 30 female patients confirms that diode laser combined with skin cooling provides a safe and effective method for long-term hair reduction in Fitzpatrick skin types IV and V. By using conservative parameters, correct hormonal workup and ultrasonography, careful patient selection and adequate gap between two sessions, gave us an excellent result with no long-term side effects and good patient satisfaction rates. To conclude, the Diode laser technique is efficient and safe in patients undergoing the elective hair reduction. But various multi-centric studies with a follow-up of more than 1 year are required to determine the long term effects of diode laser hair removal.

\section{References}

1. Courtney E, Goldberg D. Clinical Evaluation of Hair Removal Using an $810 \mathrm{~nm}$ Diode Laser with a Novel Scanning Device. J Drugs Dermato. 2016;15(11):13303.

2. Ekbäck MP, Lindberg M, Benzein E, Årestedt K. Social support: An important factor for quality of life in women with hirsutism. Health Qual Life Outcomes 2014;12(1):183.

3. Gaber M, El-Sayed S. Quality of life in patients having hirsutism. Menoufia Med J 2021;34(2):477-81.

4. Ayatollahi A, Samadi A, Rajabi-Estarabadi A, Yadangi S, Nouri K, Firooz A. Comparison of efficacy and safety of a novel 755-nm diode laser with conventional 755-nm alexandrite laser in reduction of axillary hairs. Lasers in medical science. 2020;35(2):373-8.

5. JD DJ. Clinical evaluation of hair removal using an 810 nm diode laser with a novel scanning device. Journal of drugs in dermatology. 2016;15(11):1330-3.

6. AL-Hamamy HR, Saleh AZ, Rashed ZA. Evaluation of Effectiveness of Diode Laser System (808 nm) versus Intense Pulse Light (IPL) in the Management of Unwanted Hair: A Split Face Comparative Study. Int J Med Physics, Clin Eng Radiat Oncol 2015;04(01):41-8.

7. Greppi I. Diode laser hair removal of the black patient. Lasers in Surgery and Medicine: The Official Journal of the American Society for Laser Medicine and Surgery 2001;28(2):150-5.

8. Zaleska I, Atta-Motte M. The Effectiveness of Diode Laser $805 \mathrm{~nm}$ Hair Removal in Groups of Various Ethnicity. J Med-Clin Res \& Rev 2018;2(4):1-9 